First of all we would like to thank the editor for considering our work. In the following we are giving our response to the issue raised by the editor.

Editor Comment:

Despite your efforts, we found continued overlap between the submitted manuscript and the following publication, written by you/your-co-authors.

https://doi.org/10.1016/j.robot.2018.10.005

Please rephrase any duplicated text outside the methods section. The text that needs to be addressed involves lines:

104-107

$115-121$

$131-144$

Our response:

Thanks for your deep consideration. You are right. We did as you asked and changed the mentioned above lines in the main text.

\title{
Kind regards
}

Fatemeh Ziaeetabar, Ph.D. 